\title{
NOMENCLATURAL CHANGES IN LIPARIS-COMPLEX (MALAXIDINAE, EPIDENDROIDEAE)
}

\author{
Dariusz L. SzlachetKo, Hanna B. MargońskA*, Magdalena KuŁaK \\ Department of Plant Taxonomy and Nature Conservation, Gdańsk University \\ Al. Legionów 9, 80-441 Gdańsk, Poland \\ e-mail: dokhbm@univ.gda.pl
}

(Received: May 13, 2006. Accepted: April 1, 1007)

\begin{abstract}
Explanation of taxonomic and nomenclatural problems of few taxa of Liparis sensu lato are compiled. Taxonomic status of Disticholiparis is confirmed. New nomenclatural combinations within Stichorkis are proposed.
\end{abstract}

KEY WORDS: Epidendroideae, Liparis, Cestichis, Disticholiparis, Stichorkis, Australasia

\section{INTRODUCTION}

The genus Liparis C.Rich. (Malaxidinae) is one of the most numerous representatives within Orchidaceae occurring in all continents except Antarctic. It embraces a few hundreds species. Most of them are known from tropical regions of the Old World. The species of Liparis are relatively not very variable in consideration of flower structure and generative parts and they are simultaneously characterized by a great diversity in vegetative parts structure, especially leaves. There some related genera were described in the beginning of 19th century (i.e. Lindley 1824 , 1825, 1830; Thouars 1809) which were classified within Liparis by most of botanists after that time. Some of them were restablished just in recent years i.e. Empusa Lindl. czy Cestichis Thouars. There were some difficulties with access to original works and it caused a few perturbations in formulation of some taxa. An example of such problem is the case of Stichorkis and Cestichis. The former genus Stichorkis was proposed by du Petit-Thouars in a very peculiar way and it was discussed in details by Rasmussen (1979). However Cestichis was ascribed to du Petit-Thouars incorrectly. As a matter of fact, the name appeared in Lindley's work (1824, nom. nuda, 1825, nom. leg., at sectional level, with description) and was adopted by Pfitzer (1887, nom. leg., at genus level). Later, Pfitzer (1897) corrected the erroneous genus name Cestichis, to its correct form - Stichorkis Thouars.

Rassmussen was trying to raise the nomeclatural problem of Stichorkis and Cestichis in 1979, names of the genera appeared the first time in du Petit-Thouars's work in 1809 (Fig. 1) and they were separated from Liparis. du Petit-Thouars in a very laconic diagnosis of Stichorkis put the information that the genus embraces 2 species: that propo- sed by Lamarck "Angraecum cespitosum" (=Epidendrum cespitosum Lam.) and the next one, which will be described later (Fig. 2). The latter species is Stichorkis distichis Thouars (1822, Fig. 3). Rassmusen also noted the information, but despite of it he proposed the lectotype for the former genus - Stichorkis distichis Thouars (=Malaxis disticha Thouars).

The article 10.2. ICBN (Greuter et al. 2000) proclaims that "if in the protologue of the name of a genus [...] the holotype or lectotype of one or more previously or simultaneously published species name (s) is definitely included [...] the type must be chosen [...] from among these types unless the type was indicated [...] or designated by the author of the name [...]". According to the rule presented above the type of Stichorkis may be "Angraecum cespitosum" Lam. [=Epidendrum cespitosum Lam., Fig. 4] only, because it is the only valid and existing presently species name which appears in protologue of the genus.

\section{CONCLUSIONS}

Therefore the lectotype indicated by Rasmussen (1979) must be rejected. As a consequence of this, the genus Cestichis with the same type species as Stichorkis, must be treated as the older synonym of the latter one. Simultaneously, it should be considered as a valid genus Disticholiparis Szlach. \& Marg. with Stichorkis distichis Thouars (=Disticholiparis distichis (Thouars) Marg. \& Szlach.) as the type.

\section{Stichorkis Thouars}

Nouv. Bull. Sci. Soc. Philom. Paris 1: 318. 1809.

Type: Stichorkis cespitosum (Lam) Thouars ex Marg. (Epidendrum cespitosum Lam.) 


\section{NOUVEAU BULLETIN}

\section{DES SCIENCES,}

PAR LA SOCIÉTE PHILOMATHIQUE

D E P A R I S.

\section{TOME PREMIER.}

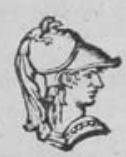

PARIS,

BERNARD, Libraire, quai des Augustins, $\mathrm{n}^{\circ} .25$.

$$
\text { M. DCCC. VII. }
$$

Fig. 1. Title page of du Petit-Thouars' 1809. Extrait de trois Memoires lus a la premiere classe de l'Institut, sur l'histoire des plantes orchidees des iles australes d'Afrique. Nouv. Bull. Sci. Soc. Philom. Paris 1.

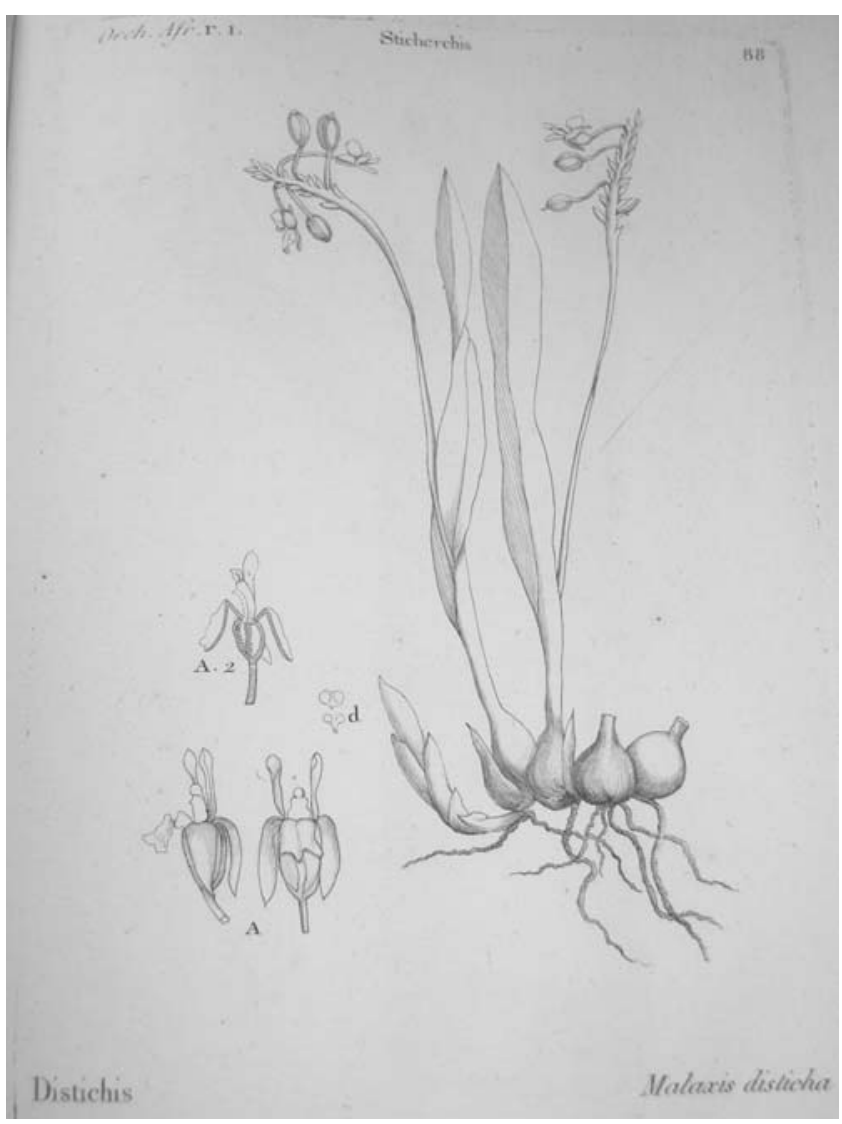

Fig. 3. du Petit-Thouars' original icone of Stichorkis distichis Thouars (1822, page 88 ).

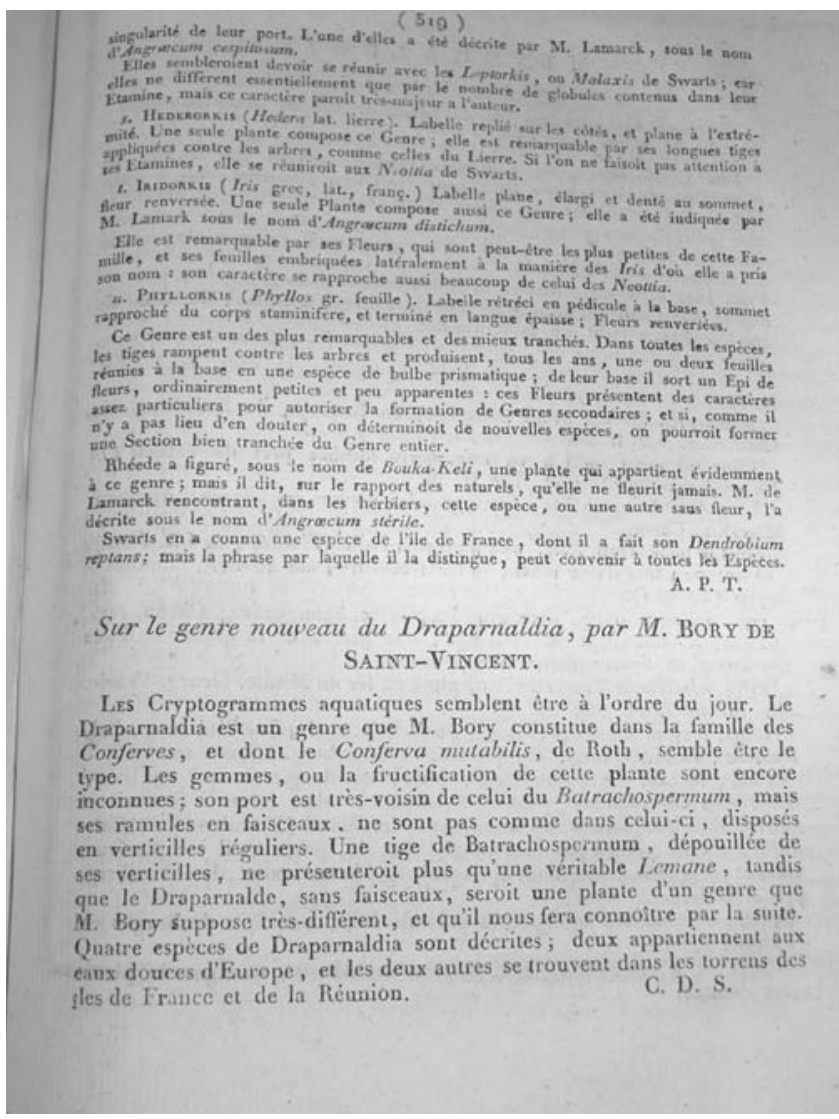

Fig. 2. The original typiphication of genus Stichorkis by du Petit-Thouars (1809, page 319).

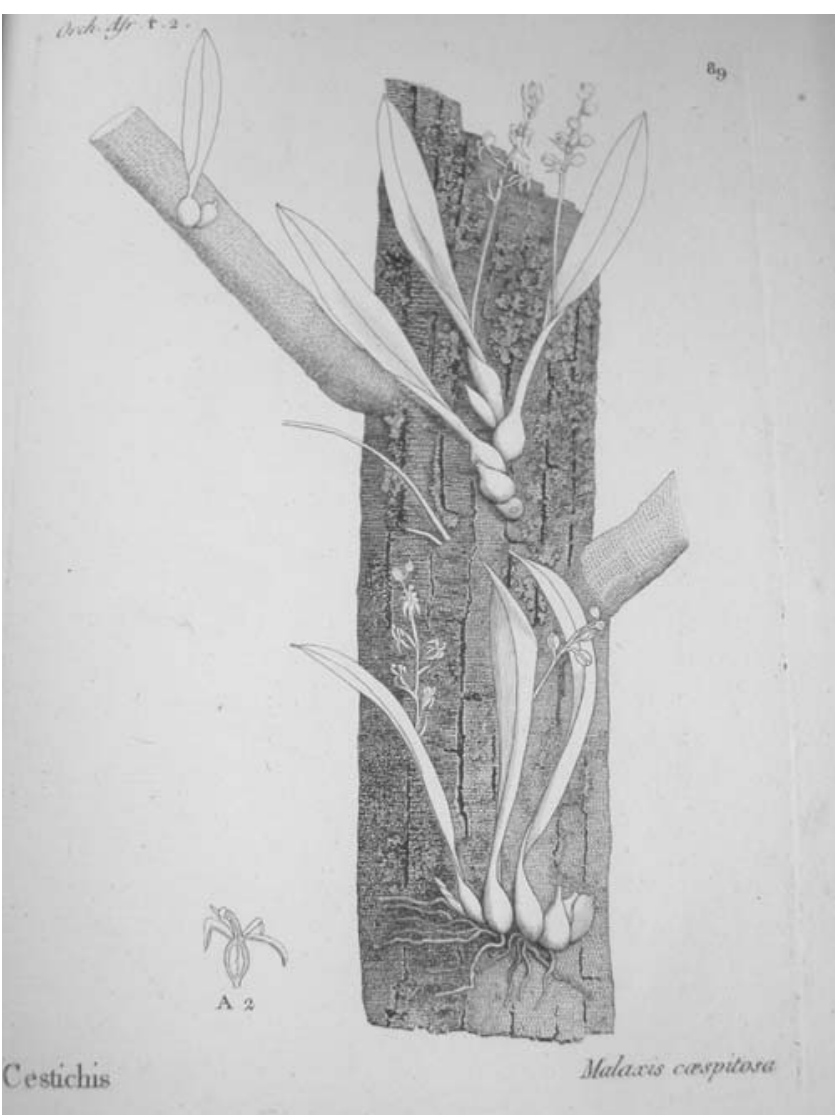

Fig. 4. du Petit-Thouars' icone of Stichorkis cestichis Thouars (Stichorkis cespitosum (Lam) Thouars ex Marg.) (1822, page 89). 
Stichorchis Thouars, Orch. Iles Austr. Afr., Prem. Tabl. Esp., Tabl. Des Generes and t. 90. 1822. orth. var. of Stichorkis.

Cestichis Lindl. ex Pfitz., Entwurf einer Naturlichen Arnordnung der Orchideen. Heidelber. 56. 1887.

The most significant features for representatives of the genus Stichorkis are epiphytic form of life and conduplicate character of leaves.

\section{NEW NOMENCLATURAL COMBINATIONS}

In consequence of the presented proofs followed, new nomenclatoral combination within genus Stichorkis are necessary:

Stichorkis acaulis (Schltr.) Marg., Szlach. \& Kułak, comb. nov.

Basionym: Liparis acaulis Schltr., Die Flora der Deutschen Schutzgebiete in der Südsee 102. 1905.

Stichorkis aptenodytes (J. J. Sm.) Marg., Szlach. \& Kułak, comb. nov.

Basionym: Liparis aptenodytes J. J. Sm., Bulletin du Jardin Botanique de Buitenzorg, Ser.3., 10: 53. 1928.

Stichorkis aurantiorbiculata (J. J. Wood \& Lamb) Marg., Szlach. \& Kułak, comb. nov.

Basionym: Liparis aurantiorbiculata J. J. Wood \& Lamb, in J. J. Wood, R. S. Beamana, J. H. Beaman, The plants of Mount Kinabalu, 2. Orchids: 243. 1993.

Stichorkis balansae (Gagnep.) Marg., Szlach. \& Kułak, comb. nov.

Basionym: Liparis balansae Gagnep., Bulletin de la Société Botanique de France 79: 165. 1932.

Stichorkis beccari (Ridl.) Marg., Szlach. \& Kułak, comb. nov.

Basionym: Liparis beccari Ridl., Journal of the Linnean Society, Botany 22: 284. 1886.

Stichorkis bicolor (J. J. Sm.) Marg., Szlach. \& Kułak, comb. nov.

Basionym: Liparis bicolor J. J. Sm., Fl. Buitenzorg, 6: 270. 1905.

Stichorkis bicuspidata (J. J. Sm.) Marg., Szlach. \& Kułak, comb. nov.

Basionym: Liparis bicuspidata J. J. Sm., Icones Bogorienses 2: 45, t. 109 C. 1903.

Stichorkis bistriata (Par. \& Rchb.f.) Marg., Szlach. \& Kulak, comb. nov.

Basionym: Liparis bistriata Par. \& Rchb.f., Transactions of the Linnean Society of London, Botany 30: 155. 1874.

Stichorkis bootanensis (Griff.) Marg., Szlach. \& Kułak, comb. nov.

Basionym: Liparis bootanensis Griff., Notulae ad Plantas Asiaticas 98.1848. (descr.) \& Not. 3: 278. 1851. (name)
Stichorkis brachystele (Ridl.) Marg., Szlach. \& Kułak, comb. nov.

Basionym: Liparis brachystele Ridl., Transactions of the Linnean Society of London, Botany 9: 162. 1916.

Stichorkis bracteata (P. E. Hunt) Marg., Szlach. \& Kułak, comb. nov.

Basionym: Liparis bracteata P. E. Hunt, North Queensland Naturalist 14 (81): 9. 1946.

Stichorkis brevicaulis (Schltr.) Marg., Szlach. \& Kułak, comb. nov.

Basionym: Liparis brevicaulis Schltr., Repertorium Specierum Novarum Regni Vegetabilis, Beihefte 1: 202. 1911.

Stichorkis chalandei (Finet) Marg., Szlach. \& Kułak, comb. nov.

Basionym: Liparis chalandei Finet, Bulletin de la Société Botanique de France 55: 338. 1908.

Stichorkis chapaensis (Gagnep.) Marg., Szlach. \& Kułak, comb. nov.

Basionym: Liparis chapaensis Gagnep. Bulletin de la Société Botanique de France 79: 166. 1932.

Stichorkis chlorantha (Schltr.) Marg., Szlach. \& Kułak, comb. nov.

Basionym: Liparis chlorantha Schltr., Repertorium Specierum Novarum Regni Vegetabilis, Beihefte 1: 201. 1911.

Stichorkis cinnabarina (J. J. Sm.) Marg., Szlach. \& Kułak, comb. nov.

Basionym: Liparis cinnabarina J. J. Sm., Bulletin du departement de l'agriculture aux Indes Neerlandaises 19: 26. 1908.

Stichorkis coelogynoides (F. Muell.) Marg., Szlach. \& Kułak, comb. nov.

Basionym: Sturmia coelogynoides F. Muell., Fragmenta Phytographiae Australiae 2: 71. 1860.

Stichorkis condylobulbon (Rchb. f.) Marg., Szlach. \& Kułak, comb. nov.

Basionym: Liparis condylobulbon Rchb. f., Hamburger Garten-Blumenzeitung 18: 34. 1862.

Stichorkis crenulata (B1.) Marg., Szlach. \& Kułak, comb. nov.

Basionym: Malaxis crenulata Blume, Bijdragen tot de flora van Nederlandsch Indië. 393.1825

Stichorkis decursiva (Rchb.f.) Marg., Szlach. \& Kułak, comb. nov.

Basionym: Liparis decursiva Rchb.f., Gardener's Chronicle \& Agricultural Gazette 2: 38. 1884.

Stichorkis dendrochiloides (Averyanov.) Marg., Szlach. \& Kułak, comb. nov.

Basionym: Liparis dendrochiloides Averyanov., Botaniceskjij Žurnal SSSR 73 (3): 432.1988. 
Stichorkis distans (C. B. Clarke) Marg., Szlach. \& Kułak, comb. nov.

Basionym: Liparis distans C. B. Clarke, Journal of the Linnean Society, Botany 25: 71, T. 29, 1889.

Stichorkis elegans (Lindl.) Marg., Szlach. \& Kułak, comb. nov.

Basionym: Liparis elegans Lindl., The genera and species of orchidaceous plants. 1830: 30 .

Stichorkis elliptica (Wight) Marg., Szlach. \& Kułak, comb. nov.

Basionym: Liparis elliptica Wight, Icones Plantarum Indie Orientalis. 5: 1735. 1852.

Stichorkis esquirolii (Schltr.) Marg., Szlach. \& Kułak, comb. nov.

Basionym: Liparis esquirolii Schltr., Fed. Rep. 12: 108. 1913.

Stichorkis fargesii (Finet) Marg., Szlach. \& Kułak Basionym: Liparis fargesii Finet, Bulletin de la Société Botanique de France, 55: 340. 1908.

Stichorkis flabellata (J. J. Sm.) Marg., Szlach. \& Kułak, comb. nov.

Basionym: Liparis flabellata J. J. Sm., Bulletin du departement de l'agriculture aux Indes Neerlandaises 19: 27. 1908.

Stichorkis fleckeri (Nicholls.) Marg., Szlach. \& Kułak, comb. nov.

Basionym: Liparis fleckeri Nicholls., North Queensland Naturalist 6 (53): 1.1938.

Stichorkis foetulenta (J. J. Sm.) Marg., Szlach. \& Kułak, comb. nov.

Basionym: Liparis foetulenta J. J. Sm. Bull. Jard. Bot. Buit. Ser.3., 2: 32. 1920.

Stichorkis gibbsiae (J. J. Sm.) Marg., Szlach. \& Kułak, comb. nov.

Basionym: Liparis gibbsiae J. J. Sm., A Contribution to the Phytogeography and Flora of the Arfak Mountains 112. 1917.

Stichorkis grandiflora (Ridl.) Marg., Szlach. \& Kułak, comb. nov.

Basionym: Liparis grandiflora Ridl., J. Bot. 22: 333. 1884.

Stichorkis grossa (Rchb.f.) Marg., Szlach. \& Kułak, comb. nov.

Basionym: Liparis grossa Rchb.f., Gard. Chron. 1: 110. 1883.

Stichorkis indifferens (J. J. Sm.) Marg., Szlach. \& Kułak, comb. nov.

Basionym: Liparis indifferens J. J. Sm., Repertorium Specierum Novarum Regni Vegetabilis 12: 24. 1913.

Stichorkis insectifera (Ridl.) Marg., Szlach. \& Kułak, comb. nov.

Basionym: Liparis insectifera Ridl., Transactions of the Linnean Society of London, Botany 9: 163. 1916.
Stichorkis kempfii (Schltr.) Marg., Szlach. \& Kułak, comb. nov.

Basionym: Liparis kempfii Schltr., Repertorium Specierum Novarum Regni Vegetabilis, Beihefte 16: 110. 1919.

Stichorkis kempteriana (Schltr.) Marg., Szlach. \& Kułak, comb. nov.

Basionym: Liparis kempteriana Schltr., Repertorium Specierum Novarum Regni Vegetabilis, Beihefte 1: 208. 1911.

Stichorkis klossii (Ridl.) Marg., Szlach. \& Kułak, comb. nov.

Basionym: Liparis klossii Ridl., Kew Bulletin. 1926: 84. 1926.

Stichorkis kinabaluensis (J. J. Wood) Marg., Szlach. \& Kułak, comb. nov.

Basionym: Liparis kinabaluensis J. J. Wood, Lindleyana 5 (2): 84. 1990.

Stichorkis kwangtungensis (Schltr.) Marg., Szlach. \& Kułak, comb. nov.

Basionym: Liparis kwangtungensis Schltr., Fed. Rep. 19: 379. 1924

Stichorkis lacerata (Ridl.) Marg., Szlach. \& Kułak Basionym: Liparis lacerata Ridl., Journal of Linnean Society of London. 22: 284. 1886.

Stichorkis latibasis (J. J. Sm.) Marg., Szlach. \& Kułak, comb. nov.

Basionym: Liparis latibasis J. J. Sm., Repertorium Specierum Novarum Regni Vegetabilis 11: 556. 1913.

Stichorkis laxa (Schltr.) Marg., Szlach. \& Kułak, comb. nov.

Basionym: Liparis laxa Schltr., Engl. Bot. Jahrb. 39: 60.1906

Stichorkis longissima (J. J. Sm.) Marg., Szlach. \& Kułak, comb. nov.

Basionym: Liparis longissima J. J. Sm., Bulletin du Jardin Botanique de Buitenzorg, Ser. 2, 13: 6. 1914.

Stichorkis luteola (Lindl.) Marg., Szlach. \& Kułak, comb. nov.

Basionym: Liparis luteola Lindl., The genera and species of orchidaceous plants. 1830: 32. 1830.

Stichorkis mannii (Rchb.f.) Marg., Szlach. \& Kułak, comb. nov.

Basionym: Liparis mannii Rchb.f., Flora 55: 275. 1872.

Stichorkis merapiensis (Schltr.) Marg., Szlach. \& Kułak, comb. nov.

Basionym: Liparis merapiensis Schltr., Botanische Jahrbücher für Systematik, Pflanzengeschichte und Pflanzengeographie 45 (104): 16. 1911

Stichorkis microblepharon (Schltr.) Marg., Szlach. \& Kułak, comb. nov.

Basionym: Liparis microblepharon Schltr., Repertorium Specierum Novarum Regni Vegetabilis, Beihefte 1: 205. 1911. 
Stichorkis monophylla (Ames) Marg., Szlach. \& Kułak, comb. nov.

Basionym: Liparis monophylla Ames, Orchidaceae 6: 294. 1920.

Stichorkis nugentiae (F. M. Bailey) Marg., Szlach. \& Kułak, comb. nov.

Basionym: Liparis nugentiae F. M. Bailey, Bot. Bull. Dept. Agric., Queensland 14: 11. 1896.

Stichorkis ovalis (Schltr.) Marg., Szlach. \& Kułak, comb. nov. Basionym: Liparis ovalis Schltr., Repertorium Specierum Novarum Regni Vegetabilis, Beihefte 1: 202. 1911.

Stichorkis pallida (B1.) Marg., Szlach. \& Kułak, comb. nov. Basionym: Malaxis pallida Blume, Bijdragen tot de flora van Nederlandsch Indië. 391. 1825.

Stichorkis parviflora (Blume) Marg., Szlach. \& Kułak, comb. nov.

Basionym: Malaxis parviflora Blume, Bijdragen tot de flora van Nederlandsch Indië 8: 392. 1825.

Stichorkis persimilis (Schltr.) Marg., Szlach. \& Kułak, comb. nov.

Basionym: Liparis persimilis Schltr., Repertorium Specierum Novarum Regni Vegetabilis, Beihefte 1: 206. 1911.

Stichorkis petelotii (Gagnep.) Marg., Szlach. \& Kułak, comb. nov.

Basionym: Liparis petelotii Gagnep., Bull. Soc. Bot. Fr. 79: 166.1932.

Stichorkis phalacrocorax (Halle) Marg., Szlach. \& Kułak, comb. nov.

Basionym: Liparis phalacrocorax Halle, Fl. N. Caled. \& Depend., 8: 282. 1977.

Stichorkis plantaginea (Lindl.) Marg., Szlach. \& Kułak, comb. nov.

Basionym: Liparis plantaginea Lindl., The genera and species of orchidaceous plants. 1830: 29. 1830.

Stichorkis pullei (J. J. Sm.) Marg., Szlach. \& Kułak, comb. nov.

Basionym: Liparis pullei J. J. Sm., Bulletin du Jardin Botanique de Buitenzorg, ser. 2, 13: 56. 1911.

Stichorkis pusilla (Ridl.) Marg., Szlach. \& Kułak, comb. nov.

Basionym: Liparis pusilla Ridl., Journal of the Linnean Society, Botany. 22: 119.1886

Stichorkis reflexa (R. Br.) Marg., Szlach. \& Kułak, comb. nov.

Basionym: Cymbidium reflexum R. Br., Prodromus Florae Novae Hollandiae 331. 1810.

Stichorkis rhombea (J. J. Sm.) Marg., Szlach. \& Kułak, comb. nov.

Basionym: Liparis rhombea J. J. Sm., Bulletin du departement de l'agriculture aux Indes Neerlandaises 43: 35. 1910.
Stichorkis scleriifolia (Schltr.) Marg., Szlach. \& Kułak, comb. nov.

Basionym: Liparis scleriifolia Schltr., Botanische Jahrbücher für Systematik, Pflanzengeschichte und Pflanzengeographie 58: 66. 1922.

Stichorkis seidenfadeniana (Szlach.) Marg., Szlach. \& Kułak, comb. nov.

Basionym: Liparis seidenfadeniana Szlach., Novon 3 (3): 302. 1993.

Stichorkis serrulata (Schltr.) Marg., Szlach. \& Kułak, comb. nov.

Basionym: Liparis serrulata Schltr., Die Flora der Deutschen Schutzgebiete in der Südsee 106. 1905.

Stichorkis spectabilis (Schltr.) Marg., Szlach. \& Kułak, comb. nov.

Basionym: Liparis spectabilis Schltr., Repertorium Specierum Novarum Regni Vegetabilis, Beihefte 1: 204. 1911.

Stichorkis stenostachya (Schltr.) Marg., Szlach. \& Kułak, comb. nov.

Basionym: Liparis stenostachya Schltr., Repertorium Specierum Novarum Regni Vegetabilis, Beihefte 1: 20. 1911.

Stichorkis stricklandiana (Rchb.f.) Marg., Szlach. \& Kułak, comb. nov.

Basionym: Liparis stricklandiana Rchb.f., Gard. Chron. 1: 232. 1880

Stichorkis swenssonii (F. M. Bailey) Marg., Szlach. \& Kułak, comb. nov.

Basionym: Liparis swenssonii F. M. Bailey, Queensland Agricultural Journal 16: 564. 1906.

Stichorkis tenulifolia (Hook.f.) Marg., Szlach. \& Kułak, comb. nov.

Basionym: Liparis tenulifolia Hook.f., Icones Plantarum 21: 2013. 1890.

Stichorkis torta (Hook.f.) Marg., Szlach. \& Kułak, comb. nov. Basionym: Liparis torta Hook.f., Icones Plantarum 21: 2014. 1890.

Stichorkis truncicola (Schltr.) Marg., Szlach. \& Kułak, comb. nov.

Basionym: Liparis truncicola Schltr., Repert. Spec. Repertorium Specierum Novarum Regni Vegetabilis, Beihefte 1: 199. 1911.

Stichorkis vestita (Rchb.f.) Marg., Szlach. \& Kułak, comb. nov.

Basionym: Liparis vestita Rchb.f., Flora 55: 274. 1872.

Stichorkis viridicallus (Holltum) Marg., Szlach. \& Kułak, comb. nov.

Basionym: Liparis viridicallus Holltum, Gard. Bull. Singapore 14: 4. 1953.

Stichorkis viridiflora (B1.) Marg., Szlach. \& Kułak, comb. nov. Basionym: Malaxis viridiflora Blume, Bijdragen tot de flora van Nederlandsch Indië. 92.1825. 


\section{ACKNOWLEDGMENTS}

We are grateful to experts Dick Brummitt, Werner Greuter, John McNeill, Gea Zijlstra and also Partick Perret for consultation and confirmation of our study results.

Special thanks for Mr. Nicolas Fumeaux $(G)$ for his help during the corresponding author's* researches. This study was financed by KBN (Polish Committee for Scientific Research) grant No. 3P04C-082-24.

\section{LITERATURE CITED}

GREUTER W., McNEILL J., BARRIE F.R., BURDET H.M., DEMOULIN V., FILGUEIRAS T.S., NICOLSON D.H., SILVA P.C., SKOG J., TREHANE P., TURLAND N.J., HAWKSWORTH D. Liparis 2000. International code of botanical nomenclature (Saint Louis Code) adopted by the Sixteenth International Botanical Congress, St Louis, Missouri, July-August 1999. Regnum Veg. 138; xviii + 474 pp.

LINDLEY J. 1824. In: S.T. Edwards, Consisting of Coloured Figures of Exotic Plants Cultivated in British Gardens; with their History and Mode of Treatment. Botanical register 10. London. Plates 825,832 .
LINDLEY J. 1825. In: S.T. Edwards, Consisting of Coloured Figures of Exotic Plants Cultivated in British Gardens; with their History and Mode of Treatment. Botanical register 11. London. Plate 882.

LINDLEY J. 1830-1840. The genera and species of orchidaceous plants. London.

PFITZER E. 1887. Entwurf einer Naturlichen Arnordnung der Orchideen. Heidelber. 108 pp.

PFITZER E. 1897. Orchidaceae. in A. Engler and K. Prantl. (eds). Die naturlichen Pflanzenfamilien Nachtrage zum II-IV Teil. Leipzig.

RASMUSSEN F.N. 1979. Nomenclatural notes on Thouars' works on orchids. Botaniska Notiser. 132: 385-391.

THOUARS du PETIT-M. A.A. 1809. Extrait de trois Memoires lus a la premiere classe de l'Institut, sur l'histoire des plantes orchidees des iles australes d'Afrique. Nouv. Bull. Sci. Soc. Philom. Paris 1: 314-319.

THOUARS du PETIT-M. A.A. 1822. Histoire particuliere des plantes orchidees recueillies sur les trois iles australes d'Afrique, de France, de Bourbon et de Madagascar. Prem. Tabl. Esp., Tabl. Des Genres and t. 90. Paris. 